\title{
Las familias transnacionales ¿una tautología? Más allá de la dicotomía “distancia/proximidad geográfica"
}

\author{
Herminia Gonzálvez Torralbo \\ Universidad Alberto Hurtado, Santiago, Chile. \\ Email: hgonzalv@uahurtado.cl
}

\begin{abstract}
Resumen: El objetivo principal del presente artículo consiste en clarificar qué se entiende por “transnacional” con relación a la familia migrante. Para lograrlo, se destacan aquellos trabajos que visibilizan las prácticas y los significados atribuidos a las posiciones de género y parentesco al interior de la familia trasnacional. Se trata de mirar "lo transnacional” con relación a la familia, más allá de la dicotomía "proximidad/distancia geográfica” sino también desde las tensiones existentes entre lo biológico y la elección en las relaciones familiares que se encuentran impactadas por la migración. Como resultado, este artículo señala que lo transnacional en relación con las definiciones de "familia transnacional” es, en muchas ocasiones, una tautología debido a que prima una mirada biologicista de las relacionas de parentesco que se deposita implícitamente, en el deseo y/o la obligación, de mantenerse vinculado.

Palabras clave: Familia transnacional, parentesco, género, migración, distancia geográfica.
\end{abstract}

\section{Transnational families, a tautology? Beyond the dichotomy geographical distance/proximity}

\begin{abstract}
This article's main objective is to provide clarity about what we understand when we talk about "transnational", regarding migrant families. To do so, we highlight those works that show the practices and meanings given to the positions of gender and kinship inside the transnational family. The goal is to look at the transnational aspect inside the family, beyond the dichotomy "geographical proximity/distance", but looking at the tensions between biology and the election of family relations which are impacted by migration. As a result, this article points out that the transnational, in relation with the definitions of "transnational family" is, in many occasions, a tautology because what comes first is the biologicistic glance of the relations of kinship, which are attributed to the wish or the obligation of keeping in touch.
\end{abstract} distance.

Keywords: Transnational family, kinship, gender, migration, geographical

\section{As famílias transnacionais, uma tautologia? Além da dicotomia “distância/proximidade geográfica”}

Resumo: O objetivo principal deste artigo é esclarecer o que se entende por "transnacional” em relação à família migrante. Para conseguir isso, salientam-se 
aqueles trabalhos que tornam visíveis as práticas e os significados atribuídos a posições de gênero e de parentesco dentro da família transnacional. Trata-se de olhar "o transnacional” em relação à família, além da dicotomia de proximidade/distanciageográfica, mas também das tensões entre o biológico e escolha nas relações familiares que são impactados pela migração. Como resultado, este artigo aponta que o transnacional com relação às definições de "família transnacional” é, em muitos casos, uma tautologia, dado que se privilegia um olhar biologicistadas relações de parentesco depositados implicitamente no desejo e/ou a obrigação de se manter vinculado.

Palavras-chave: Família Transnacional, parentesco, gênero, migração, distância geográfica.

\section{Introducción}

El creciente interés actual por la formación de las familias y hogares transnacionales ${ }^{1}$ ni es casual ni es producto únicamente de la migración femenina que busca trabajo en otros países. Tampoco responde a un único patrón migratorio: aquel caracterizado por la migración laboral del proveedor masculino de la familia. Las familias migrantes han existido en el pasado y existen en el presente, y su condición transnacional va más allá de la toma de conciencia de algunos/as investigadores/as respecto de las nuevas dinámicas y configuraciones familiares que la migración de hombres y mujeres desencadena en las familias, y en consecuencia, más allá de la lectura que en cierta forma imponen los paradigmas teóricos actuales. Pero ¿Por qué la familia transnacional está tan de moda para aquellos que estudian el fenómeno migratorio? Aunque se conocen algunos elementos que definen la familia transnacional, la tesis que se sustenta en este trabajo refiere a la falta de claridad sobre lo transnacional en el ámbito familiar apuntando a que "familia transnacional" es un concepto cuya novedad refiere principalmente a su forma familiar, pero no tanto a su contenido, el cuál es similar a cualquier definición de familia. En relación con ello, la definición de familia con la que se compara es aquella donde la familia es entendida en este comoun conjunto de relaciones interdependientes con fines de reproducción primaria en diversos ámbitos de relación individuo sociedad, tales como: socialización, seguridad, afectos, disciplinamiento, subsistencia material. Estas relaciones se organizan a través del manejo del espacio, del tiempo, del parentesco sanguíneo y/o político, el poder y la autoridad. En ello juegan una serie de recursos materiales y simbólicos que operan sobre la base de distinciones dicotómicas a modo de ejes de desigualdad: hombre/mujer, menor/adulto, sanguíneo/ político, doméstico/público, producción/reproducción, naturaleza/cultura, razón/emoción, homosexual/heterosexual, etc. (Alberdi,1999; Jelin, 1998; Yanagisako, 1979). La “distancia/proximidad geográfica” sería una dicotomía de las muchas que se incluyen dentro de la definición de familia, sim embargo ¿por qué esta dicotomía le otorgar al concepto de "familia transnacional” un estatus diferente al de familia.

La definición más utilizada de familia transnacional es la acuñada por Bryceson y Vuorela la cual señala que la familia transnacional es "Aquella 
familia cuyos miembros viven una parte o la mayor parte del tiempo separados los unos de los otros y que son capaces de crear vínculos que permiten que sus miembros se sientan parte de una unidad y perciban su bienestar desde una dimensión colectiva, a pesar de la distancia física.” (2002: 2). Además, las autoras añaden que intentar localizarlas sería un error porque son relacionales por naturaleza. Ante esta definición se podría pensar, por defecto, que hoy por hoy la mayoría de las familias migrantes son transnacionales en tanto se mantienen (de forma más o menos intensa) en relación y fomentan, en mayor o menor grado, estos vínculos con algunos de sus familiares en origen -o más países-, sean éstos aquellos que ocupan las posiciones de hijos/as, padres o tíos/as, por mencionar sólo algunas, pero ¿Qué es lo que realmente las mantiene como familia más allá de encontrarse en una situación de transnacionalidad o impactados por lo transnacional, es decir, la distancia geográfica? Esta definición, un tanto imprecisa de Bryceson y Vuorela (2002), pero a su vez muy utilizada, obliga a preguntarnos cómo estas familias "se mantienen unidas" sin explicarlo únicamente desde aquellas prácticas que permiten superar la distancia (uso de las Nuevas Tecnologías de la Información y la Comunicación-NTICs o envío de remesas). También nos interpela respecto de cómo se indaga acerca de ese "sentimiento de bienestar colectivo y de unidad" el cual se soporta más allá del vínculo de la maternidad transnacional, que es el que más se utiliza para justificar la unión de la familia cuando se encuentra dispersa por las fronteras nacionales (Gregorio y Gonzálvez 2012).

Si ponemos la mirada en la separación de los miembros de la familia como una de las partes fundamentales de casi toda definición de familia transnacional ${ }^{2}$, encontramos que detrás del discurso sobre las familias migrantes existe un debate implícito sobre la relación entre distancia y proximidad geográfica, y mantenimiento de las relaciones familiares y de parentesco. Sin embargo, este debate no es nuevo, sino que ha sido abordado por Mason (1999), y algunos aspectos del mismo han sido aplicados a su vez al estudio de la migración y el cuidado (Baldassar 2007; 2008; Baldassar, Baldock y Wilding 2007). Mason anuncia que la proximidad o la distancia geográfica es interpretada de formas distintas por las personas, es por ello que hay quienes no ven las relaciones de parentesco como significativas en sus vidas (distance-thinking), están aquellos que son capaces de tolerar y vivir en la distancia (reluctant distance thinking), y aquellos que no pueden vivir separados y demandan proximidad (local-thinking) (1999:170171). Además, el mismo autor (Mason 1999) señala otros elementos en esta relación de distancia o proximidad en las relaciones familiares que aportan un cuadro más complejo, entre ellos: la legitimidad del propósito, el género, el ánimo de los parientes especialmente de los padres cuando estos existen, la necesidad de apoyo práctico, y también el acceso a los recursos y la clase social. En efecto, no sólo la existencia de las NTICs, o la posibilidad de enviar remesas económicas explica lo que es la "familia transnacional", es algo más complejo que aquello que se puede cuantificar, pues se relaciona con las prácticas y los significados atribuidos a cada uno de los géneros y parentescos de los miembros que conforman la familia, antes, durante y después de migrar. En definitiva, 
la familia transnacional tiene como núcleo central de su definición los mismos elementos que cualquier definición de familia, siendo esto lo que pretende desarrollar a continuación.

Es por ello, que en las etnografías que he realizado sobre familias vinculadas a la migración entre Bello (Colombia) y Elche (España); entre Madrid (España) y Pereira y Medellín (Colombia) así como también en el trabajo que actualmente realizo sobre las familias en Chile $^{3}$, el impacto de la proximidad o la distancia geográfica en el mantenimiento de las relaciones familiares la he asociado tanto con familias separadas geográficamente por las fronteras que impone el Estado-nación, como con aquellas que no lo están. En ambos casos, las motivaciones que llevan a las personas a mantener el vínculo entre ellos, son similares entre quienes se movilizan cruzando las fronteras nacionales como también entre quienes que no lo hacen. Entonces ¿Qué incluye el concepto "familia transnacional” que suponga una novedad en relación con "familia”, más allá de la mera descripción de su morfología transnacional y de las prácticas transnacionales que se realizan para poder sobrellevar esta separación? ¿No son un tanto tautológicas las definiciones de familia transnacional al ser lo transnacional el elemento definidor de las mismas? ¿ंNo es lo transnacional, entendido en este caso como separación geográfica, una práctica de las muchas que conformaría una definición sustantiva de familia? Las enunciaciones existentes sobre “familia transnacional” ${ }^{4}$ ¿No deberían ir más allá de la superación de la distancia geográfica cómo su elemento constitutivo y definidor? En consecuencia, si queremos ir más lejos de lo evidente y tautológico, no tendríamos que preguntarnos ¿Qué novedad aporta "lo transnacional" con respecto a los significados de familia? ¿Aporta algo más que enfatizar la gestión de los vínculos desde la distancia?

El interés depositado en la distancia, desencadena cierta ceguera respecto de la indagación teórica (y aportes) sobre la familia transnacional, en tanto se prioriza la búsqueda bibliográfica en la literatura migratoria para explicar cómo las familias se mantienen en contacto. Esta literatura centrada, sobre todo, en la descripción de estas prácticas transnacionales, deja de lado aquellos trabajos que proceden, por ejemplo, de los estudios relacionados con familia y parentesco, los cuales, se focalizan en los significados atribuidos a las relaciones familiares y sus transformaciones y continuidades, o por ejemplo, también desconoce los trabajos centrados en las redes y el capital social. En definitiva, un diagnóstico ya señalado por Le Gall cuando afirma que "La familia transnacional generalmente ha sido silenciada dentro de la literatura de la familia contemporánea” (2005:30), disfrazada de cierta novedad desde la literatura sobre transnacionalismo pero sobre todo, el resultado, en palabras de Rivas, Gonzálvez y Gómez de un diálogo infructuoso en tanto se da "un trabajo de engarce de marcado carácter unidireccional entre los conceptos propios de este campo con aquellos relacionados con los estudios sobre familia y parentesco, es decir, desde el campo de las migraciones hacia el campo de la familia, obviando la retroalimentación que se puede dar entre ambos" (2009:28). 
Para contribuir a una mayor claridad sobre las familias transnacionales, en este trabajo se plantean cuatro momentos: En primer lugar, mostrar algunos de los antecedentes de la familia transnacional a la luz de las redes de parentesco, visibilizando aquellos aspectos relacionados con la institución familiar. En segundo lugar, examinar aquellos estudios sobre migración y familia que muestran la inclusión gradual del análisis del género y el parentesco en la migración entendidas ambas categorías como sistemas de desigualdad social inseparables, cuyas diferencias están sustentadas en la construcción social de los significados y prácticas atribuidos en tanto hombres y mujeres que, indiscutiblemente, ocupan posiciones de parentesco. En tercerlugar, revelar cómo las obligaciones morales atribuidas al parentesco se sitúan en la tensión entre la biología y la elección. Y por último,indagar por aquellos aspectos relacionados con el parentesco que no han sido considerados en los análisis que refieren a hogares y familias transnacionales, (el trabajo de parentesco), y que son necesarias para ir más allá de la dicotomía “proximidad/distancia geográfica” desnaturalizando con ello el deseo de mantener el vínculo de la "maternidad transnacional" que se le presupone a las mujeres migrantes. Este recorrido aporta al propósito fundamental de este trabajo: avanzar en la reflexión de que lo “transnacional” con relación a la familia es, en cierta forma, una tautología.

\section{Algunos antecedentes de la familia transnacional a la luz de las relaciones de parentesco}

Como antecedentes a esta temática existen una serie de trabajos que aportan claridad respecto a la formación de la condición de transnacionalidad en las familias migrantes, pero no tanto sobre aquello que la podría diferenciar de una familia entendida como tal. Dichos trabajos, contribuyen a mirar con cierta minuciosidad cómo se supera "lo transnacional en las familias", es decir, la distancia. Aunque en algunos de ellos no se menciona la palabra transnacional, sino que se habla de "familias divididas” (Murray 1981), “familias binacionales” (Curry 1992) o "familias astronautas” (Man 1995; Pe-pua et al. 1998) estos trabajos se constituyen en los pioneros con relación a las familias migrantes. Sobre algunos de ellos, se realiza un ejercicio de esclarecimiento de los aspectos que en un pasado reciente propiciaron la conformación de este tipo de familias situadas en la distancia. Asimismo, desde la historia, se puede ver cómo estas formas familiares se desencadenaron a partir de la migración laboral temporal en varias regiones del mundo (migrantes chinos en Estados Unidos, trabajadores invitados en Europa, migrantes braceros en EE.UU.), así como también producto de la migración de las élites blancas de la Europa colonial que tuvieron medios financieros para viajar y formación cultural para mantener relaciones sociales a través de largas correspondencias (Bauer y Thompson 2004).

Para comenzar, se parte del trabajo de Álvarez (1994). El autor, en su estudio sobre grupos hispanos (cubanos, mexicanos, dominicanos y puertorriqueños), establece que la familia no se puede entender aislada de su 
historia y de los vínculos actuales con sus países de origen debido al uso estratégico de la institución familiar (1994:156). Es por ello que a lo largo de su trabajo muestra, para cada uno de los casos estudiados, cuáles son los mecanismos principales a partir de los cuales estos vínculos se mantienen, destacando como elementos centrales: el parentesco, el compadrazgo, el parentesco extendido (redes) y la confianza(Álvarez 1994:156). El autor relata las especificidades de cada grupo, y a su vez, establece comparaciones de los mismos con la migración mexicana. En concreto, el parentesco extendido o las redes de relaciones con la familia extensa juegan un rol crucial en la conexión de los individuos no sólo con niveles locales y regionales sino también mas allá de las fronteras nacionales. Un ejemplo de ello, en estrecha relación con los cuidados en la migración, es cuando los abuelos viajan a EE.UU. para ayudar a sus hijos con el cuidado de los que son sus nietos/as (Álvarez 1994:157).

Otro elemento con relación al parentesco, y que es determinante para el asentamiento en destino así como la continuidad de los vínculos con origen, es la existencia de diferentes uniones conyugales. Álvarez cuenta que los tres tipos de unión realizadas por las/os dominicanos/as son: matrimonio por la iglesia, matrimonio por ley y la unión libre. Estas uniones son un elemento fundamental para adaptarse socio-económicamente a los EE.UU. puesto que la existencia de la unión libre y el matrimonio polígamo en República Dominicana, y a su vez, la aceptación de estas normas en EE.UU. conlleva que hombres y mujeres se comprometan con nuevas relaciones en el país de destino y a su vez mantengan sus vínculos familias en el país de origen (1994:157).

Asimismo, en este esfuerzo de Álvarez por mostrar las formas diversas en que la migración influye en los patrones familiares de socialización y cambio destaca, por un lado, cómo las diversas formas de viajar -coche, autobús, tren- y los precios no excesivamente caros que acompaña la migración México-EE.UU. -excepto el avión- facilita la conexión con origen. Por otro lado, el autor señala que las visitas que a menudo realizan los migrantes a sus países de origen para ver a sus parientes y disfrutar de eventos sociales y culturales que no disponen en los EE.UU. también promueve un vínculo activo, en este caso producto de la presencia. Además, la segregación residencial experimentada por las comunidades mexicanas contribuye a crear fuertes lazos étnicos y a su vez sólidas fronteras con la sociedad más amplia. Estos factores unidos a los conflictos étnicos y a la discriminación racial hacia los mexicanos ha contribuido a mantener las razones para un fuerte compromiso con instituciones socio-culturales en origen (Álvarez, 1994: 163). En definitiva, en el trabajo de Álvarez (1994) encontramos factores que se relacionan con la historia familiar previa, es decir, aspectos relacionados con la cultura familiar que permiten explicar los significados otorgados a las relaciones de parentesco, así como también, prácticas que van más allá del parentesco biológico. Todos estos elementos explican la continuidad del sentimiento de pertenencia en algunas de estas familias, los cuales, como se puede observar, van más allá de las visitas, los viajes o las remesas, es decir, van más allá de la gestión de la distancia o lo transnacional. 
En esta misma línea, el trabajo de Baca (1994), señala la importancia de los acuerdos familiares y la organización del parentesco para que la migración de origen mexicano se mantenga en EE. UU. En concreto, la autora destaca el nivel de interacción de la familia mexicana con su red de parientes (abuelos/as, tíos/as, hermanos/as casadas y sus hijos/as, compadres y padrinos), con quiénes los chicanos mantienen los vínculos de forma activa entre México y Estados Unidos (1994: 163). También, en los trabajos de Massey, Alarcón, Durán y González (1987) y Álvarez (1994) se destaca, cómo los vínculos "binacionales" se sostienen a través de los fuertes vínculos familiares ${ }^{5}$. En efecto, los trabajos de Álvarez (1994), Bacca (1994) y Massey et al. (1987), entre otros, ilustran el papel del parentesco y las redes sociales en el mantenimiento de los vínculos entre origen y destino, denominados hoy vínculos transnacionales. En definitiva, en estos trabajos se puede observar la importancia de las particularidades que cada flujo migratorio presenta, las cuales se hace necesario explicitar (historia familiar previa, significados de las relaciones de parentesco, relaciones de parentesco no bilógicas, etc.) ya que clarifican porqué se mantienen determinados vínculos familiares más allá de las posibilidades tecnológicas y monetarias existentes para resolver la distancia, las cuales se explican, sobre todo, a partir del parentesco.

\section{La consideración del género y la unidad doméstica en las redes de parentesco transnacionales}

Si bien la relevancia dada a la teoría de la red social en el examen de la migración transnacional aportó una mirada situada de las conexiones familiares y de las relaciones de parentesco que se constituyeron en un claro antecedente para el análisis de las familias transnacionales, es importante mencionar que, en un primer momento, en el estudio de esta teoría no se tomó en cuenta la influencia de las relaciones de género en la toma de decisiones a la hora de migrar (Boyd 1989; Gregorio 1998).

Aunque aparecieron trabajos que comenzaron a considerar los cambios culturales producidos por la migración, algunos de ellos centrados únicamente en la experiencia femenina (Gulati 1993; Phizacklea, 1983), poco a poco las relaciones de género fueron cobrando protagonismo (Foner 1976; 1986) tanto al interior de la unidad doméstica como con respecto a las redes sociales que se conformaban a propósito de la migración (Boyd 1989). Trabajos relevantes en este sentido fueron los de Hondagneu-Sotelo (1994) y Kibria (1993), los cuales, de forma explícita plantearon una crítica a la imagen del hogar como unidad sin jerarquías de poder y autoridad, problematizando el concepto de "estrategias familiares" para subrayar que las familias y las redes sociales formadas por los migrantes son instituciones fuertemente marcadas por el género, y en consecuencia, por los intereses que se derivan de las posiciones de género. Sin embargo, la lenta incorporación de los contextos de origen en el análisis de la configuración de los proyectos migratorios desencadenó la pérdida de información valiosa rela- 
cionada con los vínculos familiares (Bauer y Thompson 2004). En definitiva, a partir del examen de las redes sociales (teoría de la red social) en las migraciones, y posteriormente la incorporación de la perspectiva de la unidad domésticase produjo una suerte de lenta asunción del análisis del género en la migración internacional, lo cual permitió mostrar algunas de las posiciones de parentesco (mujeres, madres o abuelas, por mencionar algunas) sobre las que se soportaba el vínculo familiar en situación de separación geográfica. Así, la circulación de afectos y cuidado mencionados en los discursos de mujeres emparentadas entre sí, permitían explicar el mantenimiento de los vínculos en la distancia.

En relación con este periodo, se observa que no fueron únicamente las prácticas sociales que trataron de superar la distancia (NTICS, visitas...) las que explicaron por si solas el motivo por el cual los miembros de una familia se mantenían unidos en la distancia. También fueron importantes aquellas que reflejaron la dimensión cultural de la familia y la redes de parientes. En efecto, en el momento en el que se le dio la importancia merecida a los patrones culturales de los contextos de origen, a las redes sociales, -entre ellas, las redes de parentesco biológico o no-, y se consideraron las relaciones de género y poder para cada uno de los individuos que conformaban la unidad familiar -la perspectiva de género-, las familias y los hogares transnacionales se asumieron como un fenómeno a analizar. A partir de este momento los patrones familiares transnacionales, inicialmente los del Caribe, cobraron un gran protagonismo, debido al surgimiento de un nuevo paradigma en el análisis de las migraciones, el transnacionalismo ${ }^{6}$. Aunque el parentesco fue una categoría que operó claramente en la comprensión de estas realidades, durante la década de los 80 y los 90 hubo una mayor consciencia sobre la incorporación del análisis de género en la migración que la que hubo respecto a la incorporación del parentesco, más allá de que sin duda, ésta última categoría ocupó un papel relevante para la comprensión de la condición transnacional en las familias migrantes.

\section{La tensión entre lo biológico y la elección en las familias transnacionales}

Uno de los puntos de inflexión en el análisis de la migración transnacional es la premisa que señala que "los procesos y las relaciones de familia, entre las personas definidas como parientes, constituye el fundamento inicial para el resto de las relaciones sociales transnacionales" (Basch et al. 1994: 238). Posteriormente, Ariza matiza este supuesto señalando que "mediante sus jerarquías y vínculos de lealtad característicos, el sistema de parentesco permite que la familia (y todas las relaciones comprendidas en el vínculo consanguíneo), constituya el primer modo de organización con el que los migrantes cuentan para responder como grupo, colectivamente, a las restricciones y exigencias impuestas por el nuevo entorno de residencia” (Ariza 2002: 62). No obstante, aunque se señala que la familia es el fundamento inicial o el primer modo de organización de los 
migrantes a partir de la cual se establecen relaciones sociales transnacionales, encontramos, por un lado, que no se está diciendo nada nuevo o diferente a lo que se considera como familia, y por otro lado, que no es ni la única forma de organización social inicial de los migrantes, ni tampoco es siempre la principal, puesto que existen relaciones simbólicas (compadrazgo, padrinazgo, paisanaje o amicales) que también son relevantes en el mantenimiento de los vínculos en la distancia.

En este punto del camino, se sabe que esta premisa ha servido como inspiración para investigaciones, que enmarcadas dentro del paradigma transnacional, ponen de relieve los vínculos familiares que se dan entre aquellos miembros que se encuentran separados por fronteras nacionales. Por lo mencionado, una de las preguntas a formular sería: ¿Por qué se le da tanta relevancia a este supuesto que otorga gran protagonismo a los vínculos familiares biológicos? ¿Es posible hablar de familia transnacional desde la construcción de vínculos que no son consanguíneos? ¿Por qué hablar de familia transnacional es recurrir a la supuesta obviedad de la "consanguinidad” como elemento fundamental de la relación familiar transnacional? Como posible respuesta, se señala lo mencionado por Rivas, que es precisamente lo que parece obviarse en esta premisa: "la transnacionalización de las familias han ido generando nuevas modalidades de emparentamiento que están despojando a la biología de su carácter simbólico en la construcción del parentesco, al cuestionarla como base "natural” y factor único en la creación del mismo” (2009:13). En consecuencia, lo novedoso se relaciona con cómo la distancia geográfica genera cierta tensión entre la biología y la elección en la gestión de las relaciones de parentesco, dicotomía que se considera no en términos excluyentes sino en modalidad de coexistencia. En relación con ello, que los vínculos biológicos no son los únicos que constituyen y crean familia, es un debate que se ha puesto en evidencia a partir de la existencia deformas familiares como las maternidad por elección (por adopción o reproducción asistida) (Jociles et al. 2008; Rivas 2009; Jociles y Villamil 2012), o las familias homosexuales (Pichardo 2009), por mencionar algunas. En relación con ello, la novedad de la familia transnacional no es la separación geográfica, su novedad radica en que es una forma familiar más que viene a cuestionar el núcleo biologicista de lo que se entiende como familia.

Un ejemplo de esta tensión entre lo biológico y la elección la encontramos en el ejercicio de la maternidad transnacional, donde observamos, cómo algunas mujeres, después de más de veinte años, y después de parir en España y llevar a sus hijas a República Dominicana, no han considerado traerlas de nuevo a España porque han valorado su independencia así como también el bienestar que les podía aportar a sus hijos el medio rural y los cuidados de las abuelas (Gregorio y Gonzálvez 2012: 53). En este caso mencionado ¿no es el mantenimiento del vínculo transnacional también una consecuencia de la elección personal, y no sólo de la obligación y el deber ser que se le presupone a la relación biológica materno-filial? En este caso, superar la distancia, no es el elemento constitutivo de la preexistencia de la familia transnacional, sino también, el deseo de mantenerla. De nuevo, vol- 
vemos a la pregunta: ¿lo transnacional, cuánto aporta a la realidad de la familia migrante ${ }^{7}$. En este punto del análisis se sabe que lo transnacional no es lo novedoso, la originalidad se sitúa en la pregunta ¿cómo lo transnacional modifica los significados de lo que se entiende por familia, y en concreto, los significados (obligaciones, deberes y derechos) atribuidos a determinadas posiciones de parentesco fuertemente "naturalizadas" (maternidad)? ¿No es la familia transnacional, en la mayoría de los casos, un eufemismo de maternidad transnacional? ¿No es la familia transnacional un eufemismo de familia?

Por lo mencionado, se detecta que no se ha dicho todo respecto del mantenimiento de los vínculos familiares y de parentesco ejercidos desde la distancia, y es en este escenario donde la antropología feminista ${ }^{8}$, todavía tiene mucho que aportar. En relación con esto, las palabras de Donato, Gabaccia, Holdaway y Manalansan (2006)cobran sentido cuando en su recorrido teórico por los estudios sobre género y migración señalan, que a pesar de los esfuerzos por visibilizar el papel del género en el análisis de la migración -lo cual se observa a partir del rápido volumen de trabajos de carácter interdisciplinar surgidos desde los años 80-, la investigación sobre género no ha sido suficiente para convencer a los colegas del paradigma transnacional que esta categoría añade valor teórico al análisis migratorio. En relación con esto, si el análisis de género no ocupa el lugar que merece en el estudio de las migraciones actuales, el análisis del parentesco indisolublemente vinculado al género- todavía aparece más relegado por su “naturalización” con lo femenino (Gonzálvez 2010; 2013; Gregorio y Gonzálvez 2012). En consecuencia, las reflexiones, preguntas o aportes sobre las llamadas “familias transnacionales” todavía están por llegar. Esto se pone en evidencia especialmente a partir de la naturalización del vínculo de la maternidad transnacional, cuyas responsabilidades y elecciones personales no se conciben producto de la tensión entre lo biológico y la elección, sino más bien, producto de la naturalización de las responsabilidades transnacionales de las mujeres migrantes en lo biológico. También se hace explícito ante el hecho del desconocimiento de que el concepto de "familia transnacional” está reproduciendo los debates que el concepto más tradicional de "familia” ya superó, suponiendo en cierta forma un retroceso en los aportes necesarios sobre definiciones de familia más aperturistas e inclusivas de la diversidad.

\section{La esencialización de la maternidad en la familia transnacional: el trabajo de parentesco}

Desde los años 80, y como se ha visto en algunos de los ejemplos ubicados en los antecedentes de la familia transnacional, existe una tradición migratoria hacia Estados Unidos de mujeres mexicanas y centroamericanas en busca de trabajo remunerado como parte de un proyecto migratorio familiar. Éstas dejaron a sus hijos/as al cuidado de sus familiares, sobre todo, para el caso del patrón migratorio correspondiente a la migración 
femenina -predominante en un gran número de casos-. En esta época surgieron trabajos que fueron pioneros en el análisis de género en la migración transnacional como los de Grasmuck y Pessar (1991), Hondagneu-Sotelo (1994) y Georges (1992). Específicamente, el trabajo de Hondagneu-Sotelo y Ávila (1997), centrado en los arreglos familiares y la mediación de la maternidad en la distancia, supuso la visibilización de las relaciones familiares en un contexto transnacional. Las autoras investigaron el trabajo que realizaban las madres latinas que trabajaban en el servicio doméstico remunerado en Los Ángeles y tenían a sus hijos/as en origen. De esta forma acuñaron un nuevo término "maternidad transnacional” y sus avances apuntaron hacia dos direcciones. En primer lugar, que la transnacionalidad de la maternidad no era producto únicamente de la circularidad de la migración debido a lo efímero de estos movimientos ni se reducía a situaciones de asentamiento. En segundo lugar, definían esta maternidad ejercida en la distancia, no sólo en cuanto a la separación física entre madre e hijos/as sino también en estrecha relación con los circuitos de afecto, cuidado y apoyo financiero que transcendían las fronteras nacionales. Este trabajo de Hondagneu-Sotelo y Ávila (1997) para la migración México-EE.UU. junto con el trabajo de Gregorio (1998) para la migración entre República Dominicana y Madrid mostraron la vinculación de prácticas productivas (remesas) y reproductivas (afecto y cuidado) de carácter transnacional y a su vez, significaron el comienzo de la visibilización del papel del género y también del parentesco en la migración ${ }^{9}$. Hasta este momento, fue clave mostrar el peso específico de las relaciones de parentesco a partir de la "maternidad transnacional” como el vínculo que explicaba ese sentimiento de unidad más allá de las fronteras. Sin embargo, esta demostración, estuvo de nuevo acompañada, por el riesgo de esencializar esta relación al dar por hecho el vínculo "natural" entre las mujeres y la maternidad y entre la maternidad biológica y supuestos sentimientos maternales instintivos (Gregorio2008; Gregorio y Gonzálvez 2012: 43). De nuevo, el peso de lo biológico resurgía como explicación de la relación familiar transnacional y fundamentación de su existencia. Por lo señalado, el reto de la antropología feminista, pasó a ser la demostración de que hablar de parentesco en relación con lo transnacional implicaba un análisis que trascendía el vínculo materno-filial y la preeminencia de las relaciones familiares soportadas en los vínculos biológicos.

Posteriormente, la inclusión del enfoque de género en el paradigma transnacional consideró como relevante algo que ya habían destacado otros autores/as tiempo atrás, pero que de una u otra forma había pasado inadvertido: el trabajo de parentesco que se da al interior de cada familia, y cuyas prácticas operan de forma situada y contextualizada. En concreto, las mujeres con relación a las redes sociales han sido representadas como las encargadas del parentesco (Wetherell, Plakans y Wellman 1994:649) o como las que desempeñan lo que Micaela di Leonardo llama trabajo de parentesco, es decir, "la concepción, el mantenimiento y las celebraciones rituales a través de los lazos de parentesco dentro del grupo doméstico, incluyendo visitas, cartas, llamadas telefónicas, regalos y tarjetas recordatorias; la organización de las reuniones por vacaciones (...)” (1987:442). La considera- 
ción de este trabajo de parentesco, ejercido principalmente por mujeres, madres, hermanas o tías, muchas de ellas, migrantes, desde la mirada de la antropología feminista, supuso considerar que éste trabajo invisible no sólo se realizaba de forma altruista, sino que también existían relaciones de cuidado y apoyo que se producían de forma interesada, en tanto se desencadenaban obligaciones y responsabilidades de apoyo y cuidado en las redes de parientes (di Leonardo 1987). En consecuencia, analizar este trabajo de parentesco aplicado a la migración implicó la apertura a considerar que la maternidad transnacional no se soportaba únicamente en lo biológico, sino que iba más allá de la sangre, al ser producto también del interés personal (Gonzálvez 2010; 2013; Gregorio y Gonzálvez 2012).

En esta línea han surgido varios números especiales de revistas que, vinculan el trabajo parentesco (aunque no utilicen exactamente este concepto) con los hogares transnacionales ${ }^{10}$. En el año 2004, Chamberlain y Leydesdorff publicaron un número especial en el que exploraron el vínculo entre género y familias transnacionales a través del papel que jugaba la memoria y las narraciones en la migración, así como las subjetividades que éstas envuelven para los miembros de la familia. Este supuso una novedad, puesto que los autores se centraron en aspectos de los vínculos transnacionales que estaban relacionados con la subjetividad, emocionalidad, interioridad -aquello que no se ve-, y que contribuye a que las familias transnacionales continúen siendo familia a pesar de la distancia (Chamberlain y Leydesdorff 2004:237).

En el año 2005 se publicó toda una edición dedicada a las familias transnacionales asiáticas llamado "Transnationalizing the "Asian” family: imaginaries, intimacies and strategic intents”. Entre los contenidos del mismo se destacó cómo la obligación, la culpa y el miedo operan al interior de la familia de forma que reproducen la tradición y mantienen los vínculos comunitarios y la disciplina familiar más allá de la distancia. En este sentido, para las mujeres asiáticas la migración transnacional crea un terreno ideológico en el cual las mezclas de lealtad familiar y responsabilidad, por un lado, y la autonomía y agencia, por otro lado, son continuamente examinadas (Velayutam y Wise 2005:309). En definitiva, en estos trabajos se constata la diversidad de dimensiones a considerar en el análisis del mantenimiento del vínculo en la familia trasnacional en situación de dispersión geográfica, las cuales se relacionan con el ejercicio de ese trabajo de parentesco definido por Di Leonardo (1987).

\section{Reflexiones finales: El parentesco transnacional como desafío de la dicotomía "biología/elección”}

Como se ha podido mostrar a lo largo de este trabajo, el interés por la familia migrante se inicia a partir del fenómeno migratorio internacional contemporáneo el cual separa o dispersa geográficamente a los miembros de las familias que migran de los que no, configurando las llamadas familias 
transnacionales. Desde la década de los 80, y especialmente desde los 90, se ha producido una ingente cantidad de literatura que analiza el ámbito de lo familiar desde el prisma de lo "transnacional”. Sin embargo, en este cuerpo teórico, las preguntas más obvias han sido dejadas de lado, por ejemplo, ¿Qué aporta lo transnacional con relación a los significados sobre familia?

En este recorrido, hemos tratado de responder a esta pregunta sin caer en la pretensión de reflejar toda la literatura existentesobre migración transnacional, pero sin desconocerla.Acoger toda esta literatura iba allá de los objetivos de este trabajo, puesto que hacerlo hubiera contribuido a alimentar parte de la “miopía” existente a la hora de encontrar explicaciones a la pregunta enunciada. Demostrar el dominio del paradigma transnacional ocuparía gran parte del espacio de este artículo, dejando de nuevo sin responder aquello que se asume como obviedad.Aunque se realizó la necesaria búsqueda bibliográfica sobre la "familia transnacional”, a partir de la hipótesis del excesivo énfasis depositado en aquellas prácticas que permiten “superar” los límites geográficos que impone el Estado-nación sustentadas en la superación de la dicotomía “proximidad/distancia geográfica”, a lo largo de este trabajohemos encontrado una serie de nudos de luz que podrían apuntar hacia las respuestas que buscamos.

En efecto, encontramos que la inclusión de la crítica feminista aplicada al análisis de la movilidad transnacional ha implicado considerar otros elementos en la configuración de las relaciones de parentesco que van más allá de las obligaciones morales atribuidas a las relaciones de consanguinidad o afinidad ${ }^{11}$. La consideración del género articulado con el parentescosaca a la luz prácticas sociales significativas, que ayudan a comprender el sentimiento de pertenencia o “desapego” a la familia en aquellas personas que se encuentran en situación de lejanía física, tanto desde la interpretación deldeseo de superar la distancia y mantener los vínculos, así como también desde la elección de mantenerla separación geográfica modificando estas relaciones (la tensión mencionada entre lo biológico y la elección). Desde estas categorías, este recorrido se ha alejado del análisis de las prácticas transnacionales que superan la distancia, para con ello mostrar a la familia migrante, como una institución social con creencias, costumbres, y obligaciones situadas en determinados contextos, y sustentadas en relaciones de parentesco generizadas las cuales oscilan entre deseos altruistas o intereses personales que desencadenan unas u otras configuraciones familiares transnacionales. En definitiva, una definición de familia transnacional que es coincidente con cualquier definición de familia.

La “desconsideración” del parentesco en su relación con género, ha obviado obligaciones, derechos y deberes que son el producto no sólo de ser hombres, sino también cónyuges, abuelos o tíos, y no sólo de ser mujeres sino también madres, hermanas o tías. La inseparabilidad del género-parentesco como enfoque analítico, permite explicar el mantenimiento del vínculo familiar, no sólo a partir de elementos que van más allá del uso de las NTICs o del envío de remesas económicas y sociales ${ }^{12}$, sino también desde las responsabilidades atribuidas a estas posiciones de género-pa- 
rentesco. Esto queda claramente reflejado en los discursos de las mujeres migrantes, cuando hablan de sus responsabilidades como madres, pero también, como hermanas o hijas, lo cual, contribuye a naturalizar en los estudios migratorios, la asociación del parentesco con el "hecho femenino" de la procreación y de las relaciones de consanguinidad en la filiación (Gregorio y Gonzálvez 2012:49). El desafío no está en mostrar la centralidad de las posiciones de parentesco de las mujeres migrantes, sino las obligaciones morales derivadas de las mismas, (sobre todo monetarias), de las cuales no sólo se benefician los hijos sino también la red de parientes, en tanto "el lenguaje de las obligaciones del parentesco es orquestado por un número importante de parientes, amplificándose las obligaciones morales exigidas a las mujeres en el nuevo contexto transnacional” (Gregorio y Gonzálvez2012:50).

En definitiva, la categorías género y parentesco explican la familia transnacional, más allá de su condición transnacional o situación de separación geográfica, permitiendo comprender que la separación es una situación por la que transita, o se permanece, pero no el epítome desde el cual explicarla. Explicar lo transnacional con relación a la familia desde el argumento del deseo de mantenerse vinculado, es producto de una mirada biologicista respecto de este concepto, desde la cual se analizan las relaciones de parentesco transnacionales basadas sobre todo en las relaciones consanguíneas.Si lo transnacional quiere aportar cierta novedad al concepto de familia, debe considerar esa tensión entre lo biológico y la elección yendo más allá de la dicotomía “distancia/proximidad geográfica”, ya que de esta forma, será posible investigar otros escenarios familiares que aparecen hoy día invisibilizados producto de la esencialización que existen entre mujer y maternidad, también desde la relación familiar transnacional. En relación con ello, la familia transnacional no es más que una familia que se encuentra separada por la distancia, es por ello, que darle categoría de un concepto novedoso, y por tanto diferente al de familia, no es suficiente. 


\section{Notas}

${ }^{1}$ Para un análisis de las familias y hogares transnacionales desde un enfoque de género consultar el trabajo de Gonzálvez (2007).

${ }^{2}$ Es importante clarificar que las formas familiares transnacionales a las que nos referimos recogen la diversidad de configuraciones existentes, entre ellas, monoparentales transnacionales, extensas transnacionales y nucleares transnacionales, por mencionar algunas.

${ }^{3}$ Este trabajo es producto del diálogo entre la etnografía realizada sobre familias separadas por las fronteras del Estado-nación situadas entre España y Colombia (Gonzálvez 2010), con el trabajo de campo realizado con familias chilenas cuya separación geográfica no supera la frontera del Estado chileno (Proyecto Fondecyt Iniciación 11121245 "Las familias en Chile: El trabajo de parentesco y la generación de constelaciones familiares”-2012-2015)

${ }^{4}$ Entre las definiciones mencionadas se destacan las siguientes: "familias transnacionales encabezadas por mujeres" u hogares cuyos miembros centrales viven en al menos dos estados nación y en los cuales la madre trabaja en otro país mientras algunos o todos los que dependen de ella residen en el país originario de la migración (Parreñas 2001:361); "Familia transnacionales en hogares dispersos", entendidos como hogares que son morfológicamente definidos en términos de la distribución de sus miembros centrales en dos o más estados nación (Yeoh, Huang y Lam 2005:308); Familias transnacionales serían personas con uno o más familiares próximos viviendo en otro país (Hull et al 2010); o también refieren a "vínculos efectivos entre personas localizadas en campos sociales que incluyen dos o más países” (Bjerén 1997:237).

${ }^{5}$ La migración entre México y Estados Unidos ha sido una de la más documentada debido a la migración sostenida de trabajadores a este país.

${ }^{6}$ En palabras de Martinello y Lafleur (2008) definir el transnacionalismo como una perspectiva o concepto científico sería la elección apropiada.

7 Como señala Portes (2001; 2003) no todo lo que queda incluido dentro del paradigma trasnacional implica nuevos redescubrimientos, lo que el autor denomina "The fallacy of adumbration”.

${ }^{8}$ La antropología feminista es entendida como el examen de las desigualdades de género y parentesco, las cuales se encuentran imbricadas en determinadas esferas de la sociedad (público, privado, producción, reproducción, etc.), y se asignan de forma dicotómica a hombres y mujeres, en detrimento casi siempre de las últimas.

${ }^{9}$ Hondagneu-Sotelo y Ávila (1997)acuña el término “maternidad transnacional” y, Gregorio (1998) el concepto "grupo doméstico transnacional”.

${ }^{10}$ Las redes familiares transnacionales han sido ampliamente estudiadas por: Bryceson y Vuorela (2002); Escrivá (2000); Fouron y Glick Schiller (2001); Georges (1992); Grasmuck y Pessar (1991); Gregorio (1998); Hondagneu-Sotelo y Avila (1997);Levitt (2001); Oso (1998); Pedone (2006); Pessar (1986); Poggio y Woo (2000); Ramírez (1998); Rouse (1989) Baldassar, Baldock y Wilding (2007), Gardner y Grillo (2002), 
Rouse (1989), entre otros.

${ }^{11}$ En relación con esto, Le Gall, agrupa estos vínculos dentro de la idea de "Parentesco transnacional" refiriéndose sobre todo a los grupos de parientes que se ven impactados por la migración de algunos de los miembros de la familia (2005: 34-35), aunque no menciona, a aquellas personas que forman parte de la familia, pero que no comparten vínculos biológicos.

${ }^{12}$ Las remesas sociales han sido definidas por Peggy Levitt (2001) como las ideas, prácticas, comportamientos, identidades y el capital social que fluye entre los países de recepción y origen de los migrantes. En concreto, Levitt distingue tres tipos de remesas sociales: estructuras normativas, sistemas de prácticas y capital social. 


\section{Bibliografía}

Alberdi, Inés (1999), La nueva familia española. Taurus, Madrid.

Álvarez, Robert (1994), “Changind Patterns of Family and Ideology among Latino Cultures in the United States”. En Weaver, Thomas (ed) (1994), Handbook of Hispanic Cultures in the United States: Anthropology. Arte Publico Press Texas, Texas. Pp. 147-166.

Ariza, Marina (2002), “Migración, familia y transnacionalidad en el contexto de la globalización: algunos puntos de reflexión”. En Revista Mexicana de Sociología, 64(4): 53-84.

Baca Zinn, Maxine (1994), “Mexican-Heritage Families in the United States”. En Padilla, Félix (ed) Handbook of Hispanic Cultures in the United States: Sociology. Arte Publico Press Texas,Texas. Pp. 161-172

Baldassar, Loretta (2007), “Transnational Families and the Provision of Moral and Emotional Support: The Relationship between Truhth and Distance”. En Identities: Global Studies in Culture and Power, 14:385409.

Ídem (2008), "Debating Culture across Distance: Transnational Familues and the Obligration to Care”. En Grillo, Ralph. (ed), The family in question: immigrant and ethnic minorities in multicultural Europe. Amsterdam University Press, Amsterdam.

Baldassar, Loretta; Baldock, Cora; y Raelene, Wilding (2007), Families Caring Across Borders, Migration, Ageing and Transnational Caregiving. Palgrave Macmillan, Hampshire.

Basch, Linda; Glick Schiller, Nina y Cristina Blanc-Szanton, Cristina (1994), Nations Unbound. Transnational Projetc, Postcolonial Predicaments, And Deterritorialized Nation-States. Gordon and Breach Publishers, United Kingdom

Bauer, Elaine y Paul, Thompson (2004), “She's always the person with a very global vision: The Gender Dynamics of Migration, Narrative Interpretation and the Case of Jamaican Transnational Familiares”. En Gender and History, 16(2):334-375.

Bjerén, Gumlla (1997), “Gender and reproduction”, en Tomas. Hammar; Brochmann, Grete; Kristof, Tomas y Thomas Faist (eds). International Migration, Immobility and Development. Berg, Oxford. Pp. 219-46.

Boyd, Monica (1989), "Family and personal Networks in International Migration”. En International Migration Review, 23 (3):683-670. Special Silver Anniversary Issue: International Migration an Assessment for the 90’s (Autumn, 1989). 
Polis, Revista Latinoamericana, Volumen 15, $N^{\circ}$ 43, 2016

Bryceson, Deborah y Ulla Vuorela (2002), The transnational family: new European frontiers and global networks, Berg, New York.

Chamberlain, Mary. y Leydesdorff, S. (2004), “Transnational families: memories and narratives”. En Global Networks, 3: 227-233.

Charsley, Katharine y Alison Shaw (2006), "South Asian transnational marriages in comparative perspective”. En Global Networks, 6(4) :331-344.

Curry, Julia (1992), “Labor Migrations and Familial Responsabilities: Experiences of Mexican Women”. En Margarita B. Melville (ed.) Mexicanas at Work in the United States, University of Houston Press, Texas. pp. 47-63

Di leonardo, Micaela (1987), “The Female World of Cards and Holidays: Women, Families, and the Work of Kinship”. En Signs, 12(3):440-453.

Donato, Katharine; Gabaccia, Donna; Holdaway, Jennifer. y Martin Manalansan (2006), “A Glass Half Full? Gender in Migration Studies”. En International Migration Review, 40 (1): 3-26.

Escrivá, Ángeles (2000), “¿Empleadas de por vida? Peruanas en el servicio doméstico en Barcelona”. En Papers: Revista de Sociología, 60:327-324.

Foner, Nancy (1976), “Male and Females: Jamaican Migrants in London”. En Anthropological Quarterly, 49(1):28-35. Women and Migration (Special Issue).

Ídem (1986), "Sex roles and sensibilities: Jamaican women in New York and London”. En Simon, Rita y Caroline, Brettel (ed) International Migration: The female experience. Rowman y Allanheld, Totawa.

Fouron, Georges y Glick Schiller, Nina (2001), “All in the Family: Gender, Transnational Migration, and the Nation-State”. En Identities, 7(4):539-582.

Gardner, Katy y Grillo, Ralph (2002), “Transnational household and ritual: an overview”. En Global Networks, 2(3):179-190.

Georges, Eugenia (1992), “Gender, Class, and Migration in the Dominican Republic: Women's Experiences in a Transnational Comunnity”. En Annasl New York Academy of Sciencies. , 645:81-99.

Gonzálvez, Herminia (2007), “Familias y hogares Transnacionales: Una perspectiva de Género”. En Puntos de Vista. Cuadernos del Observatorio de las Migraciones y de la Conviviencia Intercultural de la Ciudad de Madrid, 11:7-26.

Gonzálvez, Herminia (2010), Migración colombiana, género y parentesco. La Organización social de los cuidados. Tesis Doctoral publicada, Departamento de Antropología y Trabajo Social Universidad de Granada. 
Gonzálvez, Herminia (2012), “Las Familias en Chile. El trabajo de parentesco y la generación de constelaciones familiares”. Proyecto Fondecyt Iniciación $n^{\circ} 11121245$. Comisión Nacional de Investigación Científica y Tecnológica, Santiago de Chile.

Gonzálvez, Herminia (2013a), “Los cuidados en el centro de la migración. La organización social de los cuidados transnacionales desde un enfoque de género”. En Migraciones, 33:127-153.

Gonzálvez, Herminia. (2013b), “La producción científica sobre la familia en Chile. Miradas desde la Antropología feminista”. Revista de Estudios de Género, La Ventana.

Grasmuck, Sherry y Patricia Pessar (1991), Between two islands: Dominican international migration. University of California Press, Berkeley.

Gregorio, Carmen (1998), Migración femenina su impacto en las relaciones de género. Narcea, Madrid.

Gulati, Leila. (1993), In the Absence of their men. The impact of male migration on women, Sage Publications India, London.

Hirsch, Jennifer (2003), “En el Norte la Mujer Manda” How Migration Changes Marriage”. En Hirsch, Jennifer (ed) A Courtship after Marriage. Sexuality and Love in Mexican Transnational Families. University of California Press, Berkeley.

Ho, Christine (1993), “The Internationalization of Kinship and the Feminization of Caribbean Migration: The Case of Afro-Trinidadian Immigrants in Los Angeles”. En Human Organization. 52:32-40.

Hondagneu-Sotelo, Pierrette (1994), Gendered Transitions. Mexican Experiences of Immigration. University of California Press, Berkeley.

Hondagneu-Sotelo, Pierrette y Ernestina, Avila (1997), “I'm Here, but I'm There": The Meanings of Latina Transnational Motherhood. En Gender and Society, 11( 5):548-571.

Hull, Katheleen E; Meier, Ann y Timothy, Ortyl (2010), “The Changing landscape of love and marriage”, Contexts, 9 (2): 32-37.

Jelin, Elisabeth (1998), Pan y afectos. La transformación de las familia. Fondo de Cultura Económica, España.

Jociles, María Isabel; Rivas, Ana María; Moncó, Beatriz; Villamil, Fernando y Díaz, Pablo (2008), "Una reflexión crítica sobre la monoparentalidad: El caso de las madres solteras por elección”. En Portularia: Revista de Trabajo Social, VIII, 1 265-274. 
Jociles, M.I y Villamil, F. (2012), Madres solteras por elección: representaciones sobre la fecundación sexual como vía de acceso a la maternidad. En Chungará, 44 (4), 717-731. Recuperado de http://www.scielo.cl/pdf/ chungara/v44n4/art12.pdf

Kibria, Nazli (1993), Family tightrope, Princeton University Press, Princeton.

Le Gall, Josiane (2005), “Families transnationales: bilan des recherches et nouvelles perspectives”. En Les Cahiers du Grees, 5 (1): 29-42.

Levitt, Peggy (2001), The transnational villagers. University of California Press, Berkeley.

Lin, Ge y Peter, Rogerson (1995), Elderly Parents and the Geographic Availability of their Adult Children. En Research of Aging, 17:303-331.

Man, Guida (1995), “The experience of women in Chinese immigrant families: An inquiry into institutional and organizational processes”. En Asian and Pacific Migration Journal 4 (2-3): 303-27.

Martiniello, Marco y Jean-Michel, Lafleur (2008), Towards a transatlantic dialogue in the study of immigrant political transnationalism. En Ethnic and Racial Studies, 31(4):645-663.

Mason, Jennifer (1999), “Living away from relatives: Kinship and geographical reasoning”. En MacRae Susan, (ed) Changing Britain: Families and Household in the 1990's. Oxford University Press. Oxford.

Massey, Douglas; Alarcon, Rafael, Duran, Jorge. Y Humberto, González (1987), Return to Aztlán.University of California Press, Berkeley.

Murray, Colin. (1981)., Families Divides. The impact of migrant Labour in Lesotho. Cambridge University Press, Londres.

Oso, Laura (1998), La migración hacia España de mujeres jefas de hogar. Ministerio de Trabajo y Asuntos Sociales/Instituto de la Mujer, Madrid.

Parreñas, Rachel (2001), “Mothering from a distance: emotions, gender and intergenerational relations in Filipino Transnational families”. En Feminist Studies, 27(2):361-390.

Ídem (2005), Long distance intimacy: class, gender and intergenerational relations between mothers and children in Filipino transnational families. En Global Networks, 5(4):317-336.

Phizacklea, Anne (1983), One way Ticket. Migration and female labour. Routledge \& Kegan Paul, London. 
Pedone, Claudia (2006), Tu siempre jalas a los tuyos. Estrategias migratorias y poder. Ediciones ABYA-YALA, Quito.

Pe-Pua, Rogelia; Collen. Mitchell, Sthephen. Castles, and Robyn Iredale (1998), "Astronaut families and parachute children: Hong Kong immigrants in Australia”. En Sinn, Elizabeth (ed), The last half-century of Chinese overseas, Hong Kong University Press, Hong Kong.

Pessar, Patricia (1986), “The role of gender in Dominican Settlement in the United Stated”. En Nash, June y Helen Safa (ed) Women and change in Latin America. Bergin \&Garvey Publishers, Massachussets.

Pichardo, José Ignacio (2009), Entender la diversidad familiar. Relaciones homosexuales y nuevos modelos de familia. Bellaterra, Barcelona.

Poggio, Sara y Ofelia, Woo (2000), Migracioìn femenina hacia EUA: cambio en las relaciones familiares y de geinero como resultado de la migracioìn, Edamex.. Mexico D.F.

Portes, Alejandro (2001), "Introduction: the debates and significance of immigrant transnationalism”. En Global Networks, 1(3):181-193.

Portes, Aalejandro.(2003), “Conclusion: Theoretical Convergencies and Empirical Evidence in the Study of Immigrant Transnationalism”, en The International Migration Review, 37 (3):874-892.

Ramírez, Angeles (1998), Migraciones genero e Islam mujeres marroquies en España, Agencia Española de Cooperación Internacional, Madrid.

Rivas, Ana María (2007), “Transformaciones socioculturales y cambios familiares: continuidades y rupturas”. En Lisón, Carmelo (ed), Introducción a la antropología social y cultural. Teoría, método y práctica, Akal. Madrid, pp.105-128.

Rivas, Ana M. (2009), Pluriparentalidades y parentescos electivos. En Revista de Antropología Social, 18, 7-19. Recuperado de http:// revistas.ucm.es/index.php/RASO/article/viewFile/RASO0909110007A/ 8801

Rivas, Ana M.; Gonzálvez, Herminia; y Cristina Gómez (2009), “Los Enfoques teóricos”. En Rivas, Ana María y Herminia Gonzálvez (eds) Familias transnacionales colombianas.

Transformaciones y permanencias en las relaciones familiares y de género. Catarata, Madrid.

Rouse, Roger (1989), Mexican Migration to the United States: Family relations in the development of a transnational migrant circuit. Tesis Doctoral, Standford University, Stanford. 
Polis, Revista Latinoamericana, Volumen 15, $N^{\circ}$ 43, 2016

Velayutam, Selvaraj y Amanda Wise (2005), "Moral economies of a translocal village: obligration and shame among South Indian transnational migrants”. En Global Networks, 5:27-47.

Wetherell, Charles; Plakans, Andrejs. y Barry Wellman (1994), "Social Networks, Kinshio and Community in Eastern Europe”. En Journal of Interdisciplinary History, 24(4):639-663.

Wolf, Eric (1982), Europe and the People without History. University of California Press, Berkeley.

Yanagisako, Sylvia (1979), "Family and Household: The Analysis of Domestic Groups”. En Annual Review of Anthropology, (8), pp. 161-205

Recibido: 23.07.2014

Aceptado: 30.06.2015 